\title{
BEURLING GENERALIZED PRIME NUMBER SYSTEMS IN WHICH THE CHEBYSHEV INEQUALITIES FAIL
}

\author{
R. S. HALL
}

Abstract. It is proved that there exist systems of generalized primes in which the asymptotic distribution of integers is $N(x)=$ $A x+O\left(x \cdot \log ^{-\gamma} x\right)$ with $A>0$ and $\gamma \in[0,1)$ and in which the Chebyshev inequalities

$$
\liminf _{x \rightarrow \infty} \frac{\pi(x) \log x}{x}>0, \quad \quad \limsup _{x \rightarrow \infty} \frac{\pi(x) \log x}{x}<\infty
$$

do not hold.

A nondecreasing sequence $P$ of real numbers $p_{1}, p_{2}, \cdots$ such that $p_{1}>1$ and $p_{i} \rightarrow \infty$ is called a Beurling generalized prime number system. The associated system of generalized integers, $N=\left\{n_{i}\right\}_{i=0}^{\infty}$, is the sequence of real numbers obtained by letting $n_{0}=1$ and arranging in nondecreasing order the elements of the multiplicative semigroup generated by $P$. The distribution functions $\pi(x)$ and $N(x)$ are defined in the natural way,

$$
\pi(x)=\pi_{P}(x)=\sum_{p_{i} \leqq x} 1, \quad N(x)=N_{P}(x)=\sum_{n_{i} \leqq x} 1 .
$$

Beurling [2] proved that if

$$
N(x)=A x+O\left(x \log ^{-\gamma} x\right)
$$

with $A>0$ and $\gamma>\frac{3}{2}$, then the prime number theorem holds for the system, that is, $\pi(x) \sim x / \log x$. He also showed, essentially, that there exist systems for which (1) holds with $\gamma=\frac{3}{2}$ and in which the theorem is not true. A summary of results and conjectures about systems of generalized primes is given in the recent work of Bateman and Diamond [1].

It is easy to show (as was done by the author in [3]) that if $N(x)=$ $A x+o(x)$, then

$$
\liminf _{x \rightarrow \infty} \frac{\pi(x) \log x}{x} \leqq 1, \quad \limsup _{x \rightarrow \infty} \frac{\pi(x) \log x}{x} \geqq 1 .
$$

Received by the editors November 13, 1972 .

AMS (MOS) subject classifications (1970). Primary $10 \mathrm{H} 40$.

(C) American Mathematical Society 1973 
In the following article by Diamond the Chebyshev inequalities

$$
\liminf _{x \rightarrow \infty} \frac{\pi(x) \log x}{x} \geqq a>0, \quad \limsup _{x \rightarrow \infty} \frac{\pi(x) \log x}{x} \leqq A<\infty
$$

are shown to hold when (1) is satisfied with $\gamma>1$. In this paper we show that these Chebyshev inequalities need not hold when $\gamma<1$.

TheOREM. Let $\alpha \in[0,1], \beta \in[1,+\infty]$, and $\gamma \in[0,1)$ be given. There exists a generalized prime number system in which

(i) $N(x)=A x+O\left(x \log ^{-\gamma} x\right)$,

(ii) $\lim \inf _{x \rightarrow+\infty}(\pi(x) \log x / x)=\alpha$,

(iii) $\lim \sup _{x \rightarrow+\infty}(\pi(x) \log x / x)=\beta$.

Proof. Let $\pi_{0}(x)$ be the distribution function for the rational primes. For each rational integer $n$ define the interval $I_{n}=\left(a_{n}, b_{n}\right]$ by $\log ^{1-\gamma} a_{n}=2^{n}$ and $b_{n}=a_{n} 2^{n / 4}$. Let $C_{n}$ be the set consisting of the largest $\left[(1-\alpha)\left\{\pi_{0}\left(b_{n}\right)-\pi_{0}\left(a_{n}\right)\right\}\right]$ rational primes in $I_{n}$. If $\beta<+\infty$, let $D_{n}$ be a set consisting of any $\left[(\beta-1)\left\{\pi_{0}\left(b_{n}\right)-\pi_{0}\left(a_{n}\right)\right\}\right]$ distinct real numbers in the interval $\left(b_{n}-1, b_{n}\right]$. If $\beta=+\infty$, let $D_{n}$ be a set consisting of any $\left[2^{n / 8}\left\{\pi_{0}\left(b_{n}\right)-\pi_{0}\left(a_{n}\right)\right\}\right]$ distinct real numbers in $\left(b_{n}-1, b_{n}\right]$. Let $C=$ $\bigcup_{\text {odd } n} C_{n}, D=\bigcup_{\text {even } n} D_{n}$, and let $P=\left\{p_{j}\right\}_{j=1}^{\infty}$ be the nondecreasing sequence formed from the set $P=(R-C) \cup D$, where $R$ is the set of rational primes.

Then for some $K=K(\beta)$ we have

$$
\begin{aligned}
\sum_{p \in C \cup D} \frac{\log ^{\gamma} p}{p} \leqq & \sum_{\text {odd } n}\left\{\pi_{0}\left(b_{n}\right)-\pi_{0}\left(a_{n}\right)\right\} \frac{\log ^{\gamma} a_{n}}{a_{n}} \\
& +K \sum_{\text {even } n} \frac{2^{n / 8}\left\{\pi_{0}\left(b_{n}\right)-\pi_{0}\left(a_{n}\right)\right\} \log ^{\gamma} a_{n}}{a_{n}} \\
= & O\left(\sum_{n=1}^{\infty} \frac{1}{2^{n / 2}}\right)=O(1) .
\end{aligned}
$$

Thus we see that $\prod_{p \in C}\left(1+\log ^{\gamma} p / p\right) \cdot \prod_{q \in D}\left(1-\log ^{\gamma} q / q\right)^{-1}$ converges. If for $\operatorname{Re} s>1$ we define $\left\{c_{k}\right\}_{k=1}^{+\infty}$ by

$$
\sum_{k=1}^{+\infty} \frac{c_{k}}{k^{s}}=\prod_{p \in C}\left(1-\frac{1}{p^{s}}\right) \prod_{q \in D}\left(1-\frac{1}{q^{s}}\right)^{-1},
$$

then we have

$$
\sum_{k=1}^{+\infty} \frac{\left|c_{k}\right| \log ^{\gamma} k}{k} \leqq \prod_{p \in C}\left(1+\frac{\log ^{\gamma} p}{p}\right) \prod_{q \in D}\left(1-\frac{\log ^{\gamma} q}{q}\right)^{-1}
$$


by repeated use of the inequality $\log ^{\gamma}(m n)=(\log m+\log n)^{\gamma} \leqq \log ^{\gamma} m+$ $\log ^{\gamma} n$.

Define for $\operatorname{Re} s>1$ the function

$$
\zeta(s)=\zeta_{0}(s) \prod_{p \in C}\left(1-\frac{1}{p^{s}}\right) \prod_{q \in D}\left(1-\frac{1}{q^{s}}\right)^{-1}=\sum_{n=1}^{+\infty} \frac{a_{n}}{n^{s}},
$$

where $\zeta_{0}(s)$ is the Riemann zeta function. Then $\sum_{n=1}^{+\infty} a_{n} / n^{s}=$ $\left(\sum_{m=1}^{+\infty} 1 / m^{s}\right) \sum_{k=1}^{+\infty} c_{k} / k^{s}$ and

$$
\begin{aligned}
N(x) & =\sum_{n \leqq x} a_{n}=\sum_{k \leqq x} c_{k}\left[\frac{x}{k}\right]=x \sum_{k \leqq x} \frac{c_{k}}{k}+O\left(\sum_{k \leqq x}\left|c_{k}\right|\right) \\
& =x \sum_{k=1}^{+\infty} \frac{c_{k}}{k}+O\left(x \sum_{k>x} \frac{\left|c_{k}\right|}{k}\right)+O\left(\sum_{k \leqq x}\left|c_{k}\right|\right) .
\end{aligned}
$$

From (2) we have

and

$$
\log ^{\gamma} x \sum_{k>x} \frac{\left|c_{k}\right|}{k} \leqq \sum_{k>x} \frac{\left|c_{k}\right| \log ^{\gamma} k}{k}=O(1)
$$

$$
\sum_{k \leqq x}\left|c_{k}\right| \leqq O(1)+\frac{x}{\log ^{\gamma} x} \sum_{k \leqq x} \frac{\left|c_{k}\right| \log ^{\gamma} k}{k}=O\left(\frac{x}{\log ^{\gamma} x}\right) .
$$

Thus $N(x)=x \sum_{k=1}^{+\infty} c_{k} / k+O\left(x / \log ^{\gamma} x\right)$.

We next show that (ii) and (iii) hold. Since $\pi_{0}(x) \sim x / \log x$ we have

$$
\lim _{n \rightarrow \infty} \frac{\pi_{0}\left(a_{n}\right)}{\pi_{0}\left(b_{n}\right)}=0 \text { and } \quad \lim _{n \rightarrow \infty} \frac{\pi_{0}\left(b_{n-1}\right)}{\pi_{0}\left(a_{n}\right)} 2^{n / 8}=0 .
$$

From the fact that

$$
\begin{aligned}
\pi(x) & \geqq \pi_{0}(x)-\sum_{b_{n} \leqq x ; n \text { odd }}\left[(1-\alpha)\left\{\pi_{0}\left(b_{n}\right)-\pi_{0}\left(a_{n}\right)\right\}\right] \\
& \geqq \pi_{0}(x)-(1-\alpha) \pi_{0}(x)
\end{aligned}
$$

it is clear that

$$
\liminf _{x \rightarrow+\infty} \frac{\pi(x) \log x}{x}=\liminf _{x \rightarrow+\infty} \frac{\pi(x)}{\pi_{0}(x)} \geqq \alpha .
$$

On the other hand

$$
\begin{aligned}
\pi\left(a_{n}\right) & \leqq \pi_{0}\left(a_{n}\right)+K \sum_{k<n ; k \text { even }} 2^{k / 8}\left\{\pi_{0}\left(b_{k}\right)-\pi_{0}\left(a_{k}\right)\right\} \\
& \leqq \pi_{0}\left(a_{n}\right)+K 2^{n / 8} \pi_{0}\left(b_{n-1}\right)
\end{aligned}
$$


It follows then that $\lim _{n \rightarrow \infty} \pi\left(a_{n}\right) / \pi_{0}\left(b_{n}\right)=0$ and we have (i), since

If $\beta<\infty$, then

$$
\begin{aligned}
\underset{x \rightarrow \infty}{\liminf \frac{\pi(x)}{\pi_{0}(x)}} & \leqq \liminf _{n \rightarrow \infty ; n \text { odd }} \frac{\pi\left(b_{n}\right)}{\pi_{0}\left(b_{n}\right)} \\
& =\liminf _{n \rightarrow \infty ; n \text { odd }} \frac{\pi\left(a_{n}\right)+\alpha\left\{\pi_{0}\left(b_{n}\right)-\pi_{0}\left(a_{n}\right)\right\}}{\pi_{0}\left(b_{n}\right)}=\alpha .
\end{aligned}
$$

$$
\pi(x) \leqq \pi_{0}(x)+\sum_{b_{n} \leqq x ; n \text { even }}(\beta-1)\left\{\pi_{0}\left(b_{n}\right)-\pi_{0}\left(a_{n}\right)\right\} \leqq \beta \pi_{0}(x) .
$$

Similarly, it follows that

$$
\begin{aligned}
\limsup \frac{\pi(x)}{\pi_{0}(x)} & \geqq \limsup _{n \rightarrow \infty ; n \text { even }} \frac{\pi\left(b_{n}\right)}{\pi_{0}\left(b_{n}\right)} \\
& =\limsup _{n \rightarrow \infty ; n \text { even }} \frac{\pi\left(a_{n}\right)+\beta\left\{\pi_{0}\left(b_{n}\right)-\pi_{0}\left(a_{n}\right)\right\}}{\pi_{0}\left(b_{n}\right)}=\beta .
\end{aligned}
$$

Thus, $\lim \sup _{x \rightarrow \infty} \pi(x) \log x / x=\beta$. If $\beta=\infty$, then

$$
\begin{aligned}
\limsup _{x \rightarrow \infty} \frac{\pi(x)}{\pi_{0}(x)} & \geqq \limsup _{n \rightarrow \infty} \frac{\pi\left(b_{n}\right)}{\pi_{0}\left(b_{n}\right)} \\
& =\limsup _{n \rightarrow \infty} \frac{\pi\left(a_{n}\right)+\left(2^{n / 8}+1\right)\left\{\pi_{0}\left(b_{n}\right)-\pi_{0}\left(a_{n}\right)\right\}}{\pi_{0}\left(b_{n}\right)}=\infty .
\end{aligned}
$$

This completes the proof.

A slightly more complicated argument shows that (ii) and (iii) can hold for systems in which $N(x)=A x+O(x / g(x))$ provided $g(x)=o(\log x)$. Details may be found in [3].

\section{REFERENCES}

1. P. T. Bateman and H. G. Diamond, Asymptotic distribution of Beurling's generalized prime numbers, Studies in Number Theory, vol. 6, Math. Assoc. Amer., PrenticeHall, Englewood Cliffs, N.J., 1969, pp. 152-210. MR 39 \#4105.

2. A. Beurling, Analyse de la loi asymptotique de la distribution des nombres premiers généralisés. I, Acta Math. 68 (1937), 255-291.

3. R. S. Hall, Theorems about Beurling's generalized primes and the associated zeta function, Ph.D. Thesis, University of Illinois, Urbana, Ill., 1967.

Department of Mathematics, Willamette University, Salem, Oregon 97301 